\title{
A COMPARISON OF LASER AND STRUCTURED LIGHT SCANNING TECHNOLOGIES FOR ARCHAEOLOGICAL APPLICATIONS
}

\author{
Nikolaos Papas ${ }^{1}$, Konstantinos Tsongas ${ }^{1}$, Dimitrios Karolidis ${ }^{2}$, Dimitrios Tzetzis $^{1}$ \\ ${ }^{1}$ Digital Manufacturing and Materials Characterization Laboratory, School of Science and Technology, International \\ Hellenic University, 14km Thessaloniki, 57001 N. Moudania, Greece \\ ${ }^{2}$ Archeological Museum of Thessaloniki, 6 Manoli Andronikou Street, 54621 Thessaloniki, Greece
}

Corresponding author: Dimitrios Tzetzis, d.tzetzis@ihu.edu.gr

\begin{abstract}
Reverse engineering and in particular threedimensional digitization have become an essential part of the documentation of archaeological findings. 3D scanning produces a high-precision digital reference document. The factors that influence the quality of the 3D scanned data are the scanned object's surface colour, its glossiness and geometry, and the ambient light during the scanning process. However, the actual equipment and scanning technologies are of primary importance. The current paper presents a qualitative and quantitative comparison between two 3D scanning devices of different technologies; structured light 3D scanning and laser 3D scanning. The benchmark for this comparison is an ancient Roman vase from the city of Thessaloniki, Greece. The object was scanned with every possible setting on each scanner, but only one configuration of settings on each device was selected for the final comparison. The main criterion for the final selection of the two 3D models acquired with the use of the two technologies was the proximity in the number of points and polygons produced for digitally restoring the ancient vase in the best possible way. The results indicate important differences regarding the accuracy of the final digital model. The laser technology produced better accuracy but with a significant cost in scanning time and model data size. On the other hand, the structured light technology achieved the optimal combination of scanning quality and accuracy, along with reduced acquisition time of scan data.
\end{abstract}

Key words: structured light, laser 3D scanning, digital restoration, digital archaeology, Reverse Engineering.

\section{INTRODUCTION}

In scholarly archeological research, the application of reverse engineering for documenting ancient artifacts and monuments is demonstrated in numerous case studies [110]. In particular, 3D scanning is widely used in areas of digital documentation, shape analysis, visualization, and digital restoration, while the entire archaeological reconstruction pipeline has been presented by other researchers [11,12]. Other surveys have been also focused on digital preservation of cultural heritage [13-
16], which emphasized the difficulties that have to be overcome for scanning complex geometries. The preparatory phase of the digitizing process includes the selection and proper combination of the scanner's capabilities after an optimization procedure. The current paper presents a detailed comparison between two different 3D scan technologies: structured light 3D scanning and laser 3D scanning. The object used as a benchmark for the comparison was a two-handled cup that was found during the excavations in the Galerius palace that is located in the center of Thessaloniki, a town in northern Greece. The cup was originally used for drinking wine and is dated from the late $1^{\text {st }}-$ early $2^{\text {nd }}$ century $\mathrm{AD}$. One of the two handles and a big part of its body and rim are missing. The comparison between the two scanning technologies covers all the production stages of the final 3D model, beginning from the acquisition of the scan data, going through the manipulation of the point cloud, and concluding to the final meshing algorithms provided from the firmware of each device. Finally, no supplementary software was used for the processing of the $3 \mathrm{D}$ scanned data.

\section{SCANNING PROCEDURE}

The scanning procedure of the vase took place at the conservation laboratory of the Archaeological Museum of Thessaloniki (AMTh). The object used as a benchmark for the comparison of the two scanning technologies was a two-handled cup dating from the late $1^{\text {st }}$ - early $2^{\text {nd }}$ century $\mathrm{AD}$, as portrayed in Fig. 1.

It was found during the excavations in the Galerius palace that is located in the center of Thessaloniki and it was used for drinking wine.

The criteria that led to the choice of the specific vase were its compact size and the fact that more than half of it was missing. The last one was deemed important because a significant number of random surface edges, that were ideal for optical testing of the scanning quality, were exposed. 

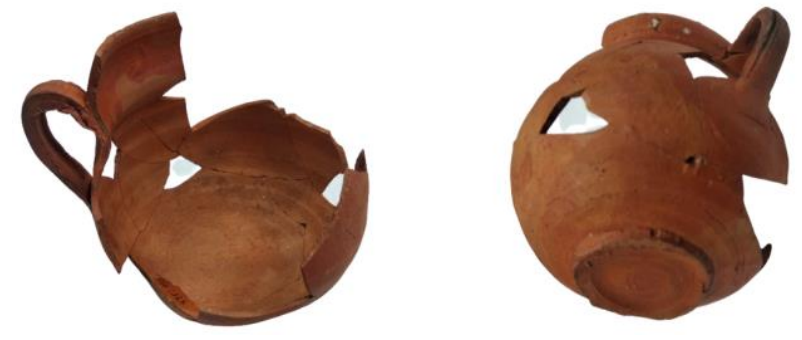

Fig. 1. Ancient vase used as a benchmark

The ancient vase was scanned with every possible configuration of each apparatus. The point cloud of each configuration was processed with the firmware of each apparatus to create a triangular mesh. The basic steps of point cloud processing included the subtraction of points captured from the surrounding environment and the removal of excess points located in the proximity of the geometry volume. The latter process is automatic and thus based on the specific capabilities of each firmware. To validate the comparison, some preestablished requirements were met as much as possible. A $360^{\circ}$ rotational scan was selected with the same number of rotations for each configuration.

Specifically, two different $360^{\circ}$ rotational scans were acquired from each apparatus. The first scan acquired the basic geometrical information and was implemented by placing the object in its physical position. The second scan was implemented by fixing the object in a slightly tilted position in order to capture the interior geometry, and more important, to capture the details on the edges located in the regions of the missing segments.

Each mesh created was left as it was originally acquired with no further processing because the goal of this research is the comparison of the default point cloud acquisition and the default meshing algorithms. It should be noticed here that the advanced capabilities of the available software in mesh post - processing are not amongst the goals of the present research.

\subsection{D laser scanning}

Initially, the vase was scanned with the use of the Next Engine ${ }^{\mathrm{TM}}$ laser scanner. The specifications of this apparatus are demonstrated in Table 1.

Table 1. Next Engine ${ }^{\mathrm{TM}}$ laser scanner specifications

\begin{tabular}{|c|c|c|}
\hline Parameter & Value & Units \\
\hline Field depth & $130 \times 90$ & $\mathrm{~mm}$ \\
\hline Accuracy & 0.127 & $\mathrm{~mm}$ \\
\hline Resolution & 40,000 & points $/ \mathrm{in}^{2}$ \\
\hline
\end{tabular}

Nine different scanning configurations were used that produced nine different meshes. Initially, one can select between three scanning qualities, Standard (MD), Quick (SD) and Fine (HD) which correspond to the points per square inch that will be acquired. During the fusion process one can select between three different meshing quality algorithms: low, medium, and high. The algorithms correspond to the number of triangles that will be generated in order to capture the geometrical details of the object with low being the lowest number of triangles. The statistics regarding the number of points, polygons, scanning time, and file size of the final STL model for each of the nine different configurations are demonstrated in Figures 2(a), 2(b), 2(c) and 2(d), respectively.

\subsection{D structured light scanning}

The scanning device used was a Scan in a Box ${ }^{\mathrm{TM}}$ structured light scanner. The specifications of this device are offered in Table 2.

Table 2. Scan in a Box ${ }^{\mathrm{TM}}$ structured light scanner specifications

\begin{tabular}{|c|c|c|}
\hline Parameter & Value & Units \\
\hline Field depth & $100 \times 80$ & $\mathrm{~mm}$ \\
\hline Accuracy & 0.08 & $\mathrm{~mm}$ \\
\hline Resolution & 100,000 & points $/$ in $^{2}$ \\
\hline
\end{tabular}

By selecting a specific field of view, a default quality of the scanned object is already predetermined. The final output quality that is obtained is a combination of the field of view and of the meshing algorithms that are used during the processing of the point cloud. There are four different meshing algorithms: design object, technical object, sculpture, and small artistic object. The first two are not suitable for the vase scanned in this study and are only included for reference purposes in the statistics of this research.

Small artistic object achieves the highest detail level and precision of the mesh with no limits in the number of triangles that could be created. Sculpture achieves high detail and precision but with lower tolerance resulting in a more confined number of triangles.

Another parameter that is taken into account is the smoothing of the final model during the meshing procedure. Three different smoothing parameters were used: 1.0, 1.5, and 2.0. This specific smoothing parameter is inversely analogue to the final meshing quality, meaning that a lower number of smoothing produces higher number of triangles and polygons. 


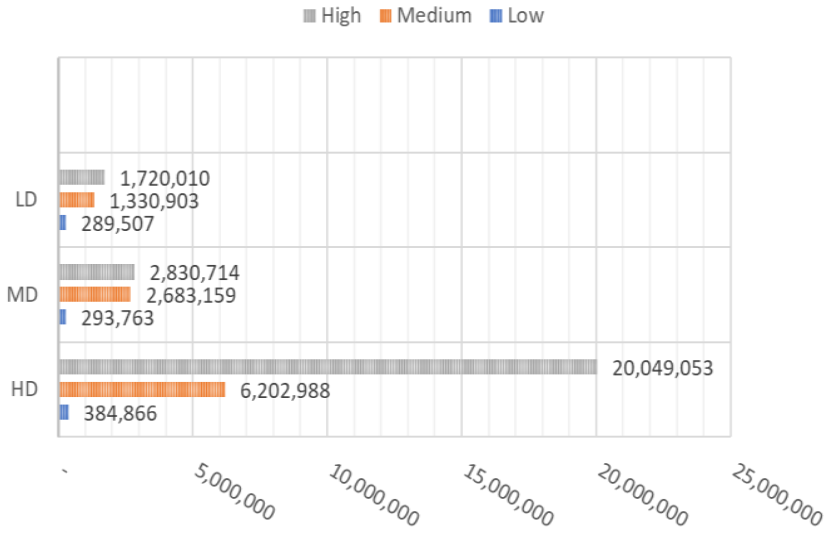

(b)

(a)

FILE SIZE (MB)

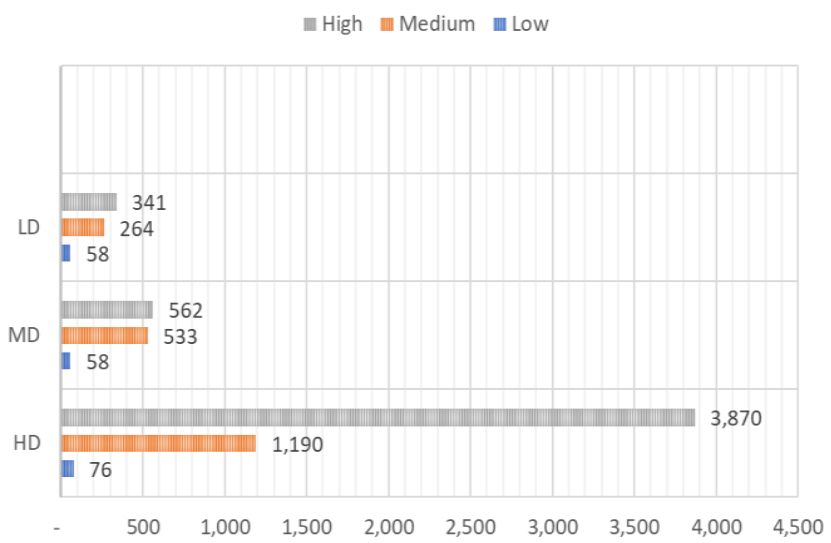

(c)

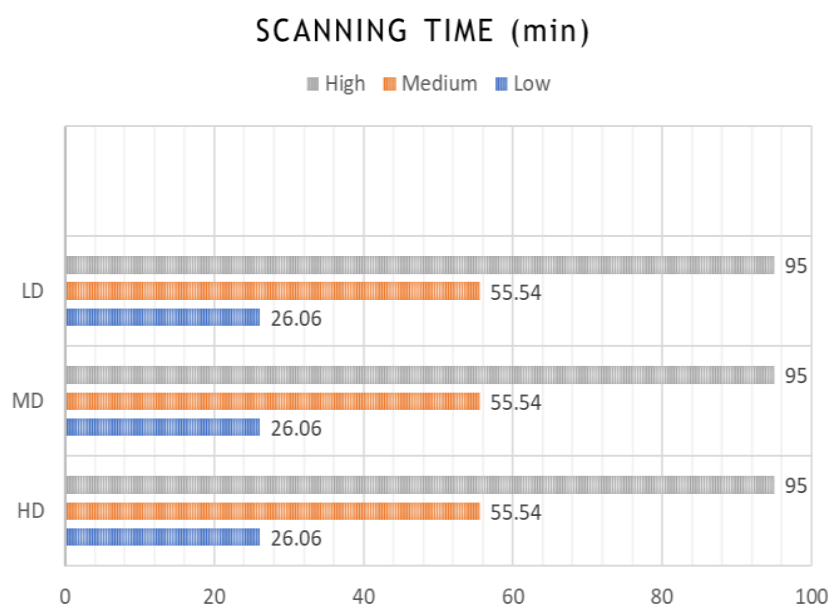

(d)

Fig. 2. Laser scanning: (a) Number of points acquired for each configuration, (b) Number of polygons acquired for each configuration, (c) File size of final STL model for each configuration and (d) Scanning time required for each configuration

The three smoothing parameters were selected experimentally. It was observed that a smoothing parameter lower than 1.0 had little to no effect in the number of triangles created and thus to the quality of the final 3D model while a smoothing parameter higher than 2.0 did not meet the quality specifications that were set initially. The statistics regarding the number of points, polygons, scanning time and file size of the final STL model for each of the six different configurations are demonstrated in Figures 3(a), 3(b), $3(c)$ and 3(d), respectively.

\section{RESULTS AND DISCUSSION}

The laser scanning technology produced nine 3D models, while the structured light technology produced six. An a priori correspondence of the configuration on the two devices is not possible, thus the comparison is solely based on the results obtained. Two models, one from each technology, were selected for comparison. The basic criterion for the selection was the proximity in polygons created during the execution of the meshing algorithm. An approximate number in polygons translates as the capability of the two algorithms to produce similar geometric accuracy. With the specific selection, the comparison was focused on the ability of each firmware to process a specific number of points. Table 3, presents the two models from each technology with the highest proximity in the number of polygons and the configuration that corresponds to each model.

The final 3D models that were chosen and produced from the two-parameter configurations can be seen in Figure 4. Figure 4(a) and Figure 4(b) show the 3D final model of the ancient vase acquired from structured light and laser technologies respectively. Figure 4(c) and Figure 4(d) show another perspective of the 3D model acquired from structured light and laser technologies, respectively.

Figures 5(a) and 5(b) focus on a specific region of the digitized ancient vase to illustrate the quality of an edge capture, which is commonly a problematic region during the scanning process.

Figures $6(\mathrm{a})$ and $6(\mathrm{~b})$ are a comparison of the 3D model created by the structured light technology and the physical object. A focus at a specific segment of the object presents the level of detail that was achieved for the internal geometry of the artifact and 
for the specific configuration of the 3D scanner. It is reminded that, though the result is adequately satisfying, it is not the highest level of detail that was achieved but the level of detail that was selected for the comparison of the two scanning technologies. In general, the objective, when digitizing an object, is to achieve an accuracy that is dictated by the requirements of the final use of the 3D model.

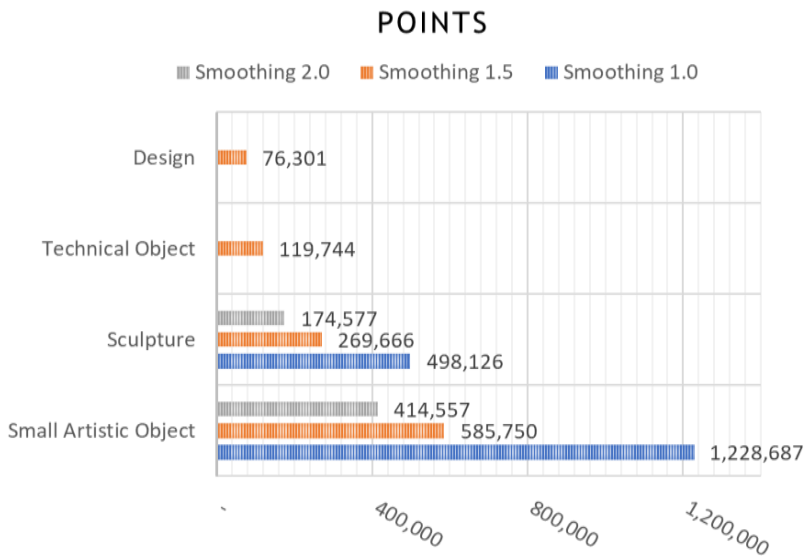

(a)

FILE SIZE (MB)

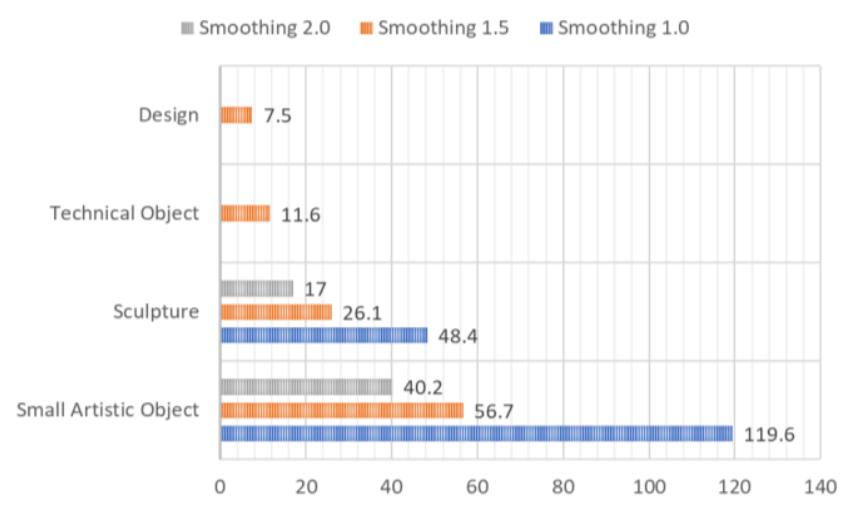

(c)
Table 3. Comparison of the two models with the proximity in polygons

\begin{tabular}{|c|c|c|}
\hline \multicolumn{3}{|c|}{ RESULTS } \\
\hline Scanning technology & Structured light & Laser \\
\hline Points & 498,126 & 913,000 \\
\hline Polygons & 990,753 & $1,330,903$ \\
\hline Scanning time (min:sec) & $11: 20$ & $26: 06$ \\
\hline File size (MB) & 48.4 & 264 \\
\hline Configuration & $\begin{array}{c}\text { Sculpture } \\
\text { Smoothing 1.0 }\end{array}$ & $\begin{array}{c}\text { LD } \\
\text { Medium }\end{array}$ \\
\hline
\end{tabular}

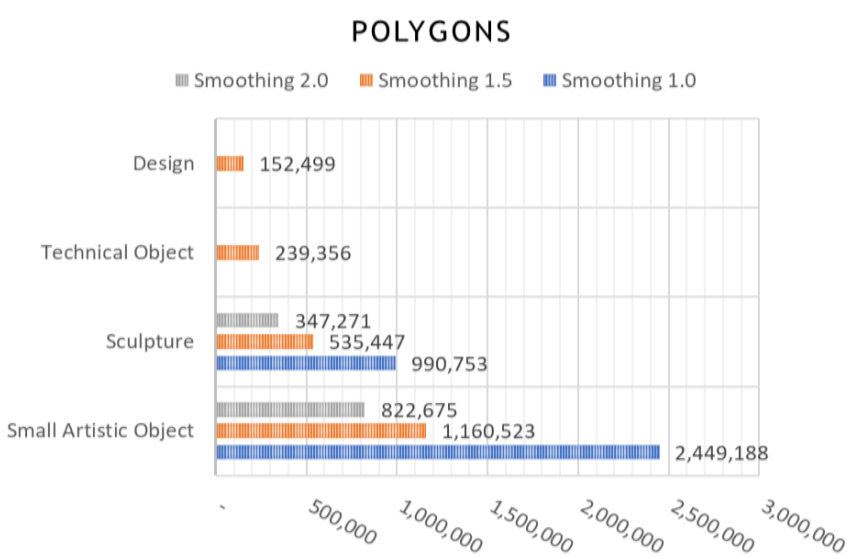

(b)

SCANNING TIME (min)

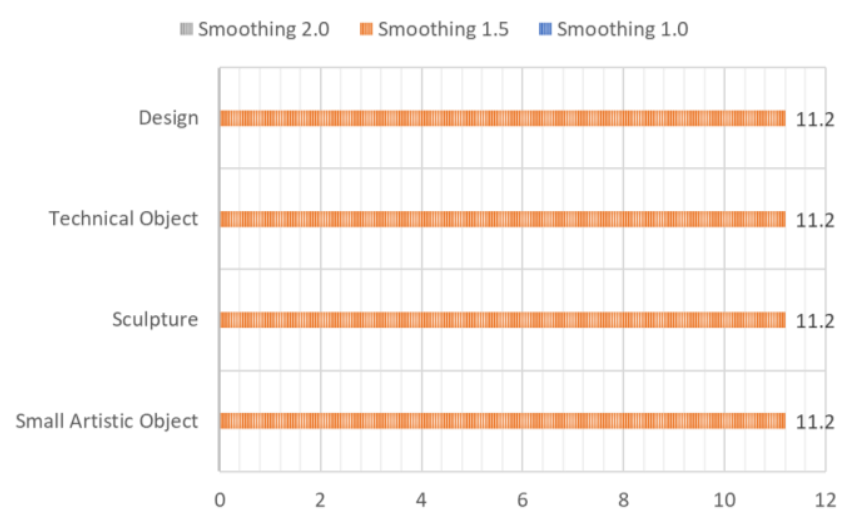

(d)

Fig. 3. Structured light scanning: (a) Number of points acquired for each configuration, (b) Number of polygons acquired for each configuration, (c) File size of final STL model for each configuration and (d) Scanning time required for each configuration

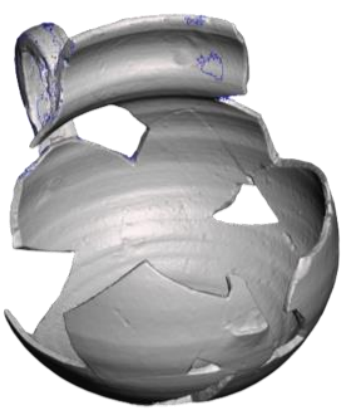

(a)

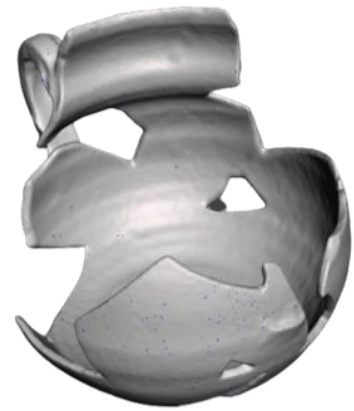

(b)

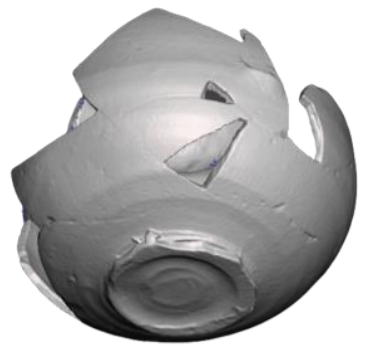

(c)

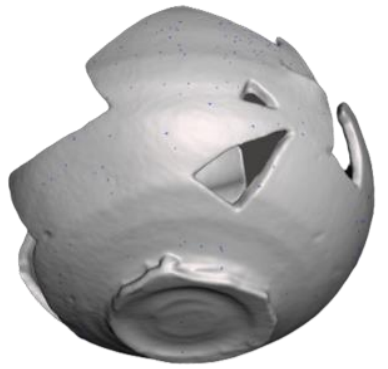

(d)

Fig. 4. Final 3D model from both scanning technologies (a) structured light top view, (b) laser scanning top view, (c) structured light bottom view and (d) laser scanning bottom view 


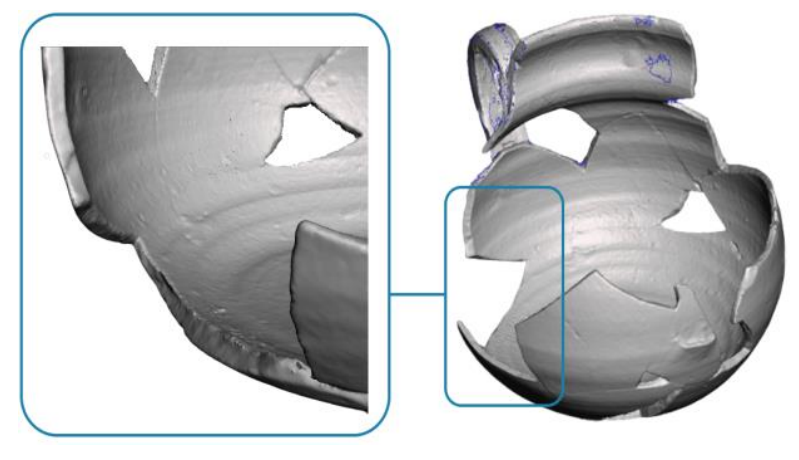

(a)

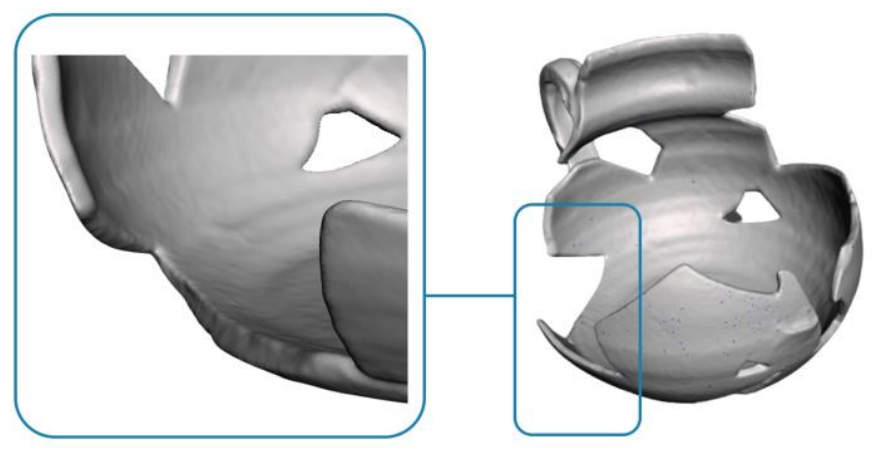

(b)

Fig. 5. 3D model derived from (a) structured light technology and (b) laser scanning technology, with detailed capture of specific edges

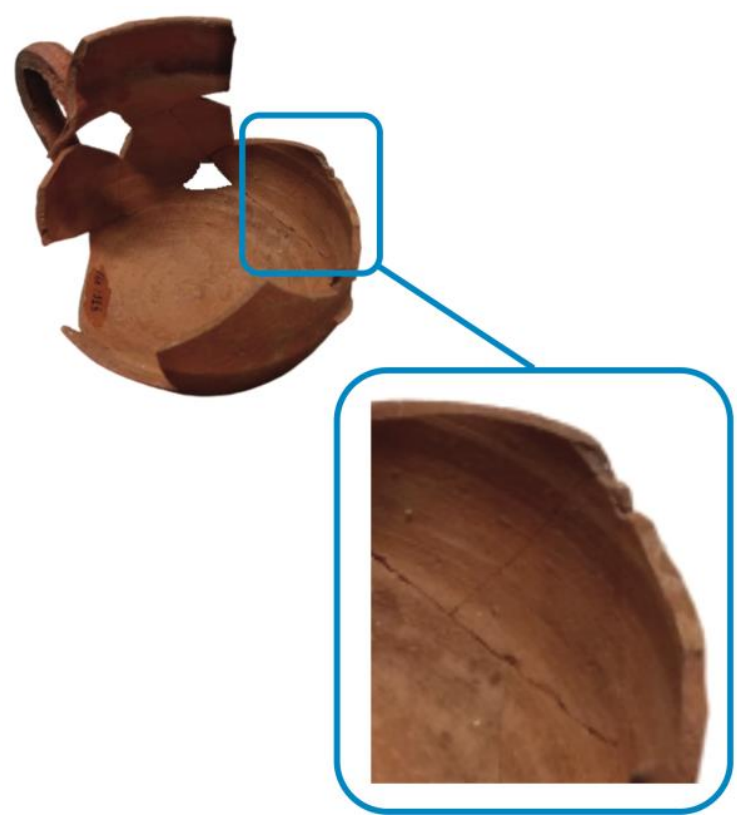

(a)

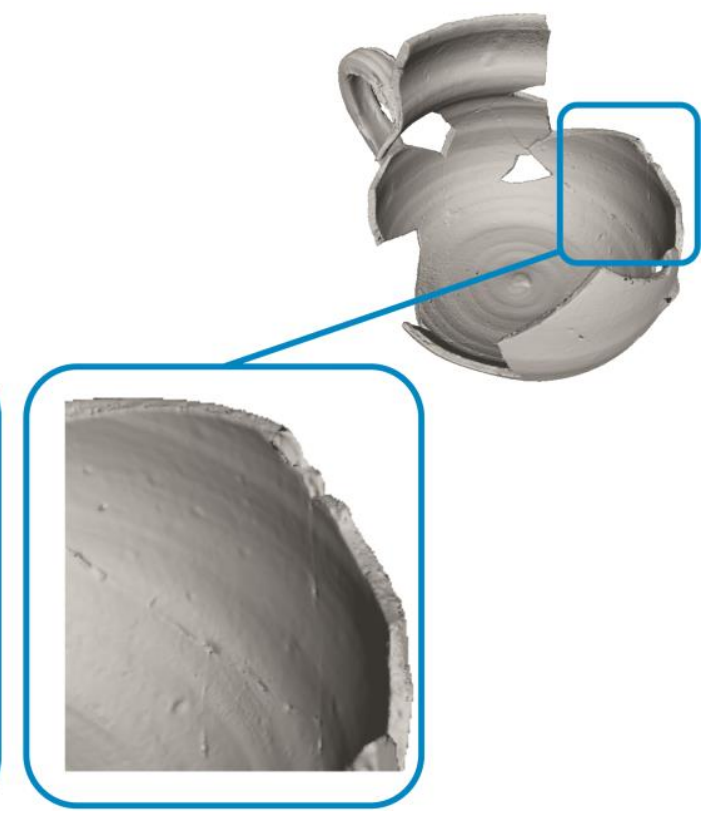

(b)

Fig. 6. Comparison between (a) physical object and (b) 3D model created from structured light technology

\section{CONCLUSIONS}

The findings of this study illustrate that the laser scanner can achieve the highest accuracy possible and can also acquire information from less accessible areas of the original object. The structured light scanner can produce a model of almost equal accuracy but at a significantly shorter time. This comparison of the two selected models has revealed that for a mesh of a predefined and approximate number of polygons, the structured light technology requires a smaller number of acquired points and thus a significantly shorter scanning time and data size of the final model. The mesh created is more detailed, compared to the actual ancient vase, but with a larger number of topological errors throughout the mesh. The mesh created from the laser scanner is less detailed compared to the actual vase, but the topology of the final model has almost no errors.
Overall, the laser scanner can produce a final STL model of higher accuracy but with a significant drawback in the scanning time and data size of the final model. In the end, with the use of a structured light scanner, one can achieve similar accuracy with less than half of the scanning time, while choosing a meshing algorithm that can significantly reduce the model size.

\section{ACKNOWLEDGEMENTS}

The project e-SKAPANI: "Thessaloniki during the Galerius era - Reviving a glorious historical period of the city, guided by interdisciplinary research and cutting-edge technologies" is supported and funded by E.U. and the National Action "Special Actions" - "Open Innovation in Culture", under the 
operational program "EPAnEK 2014-2020 Competitiveness - Entrepreneurship - Innovation" (Project Number: Т6YВП-00202).

\section{REFERENCES}

1. Chatzikonstantinou, A., Tzetzis, D., P. Kyratsis, P., Bilalis, N., (2014). Replica Fabrication of a Greek Paleontological Find Utilizing Laser Scanning and Fused Deposition Modeling, AMM, 657, 795-799.

2. Koumartzis, N., Tzetzis, D., Kyratsis, P., Kotsakis, R.G., (2015). A New Music Instrument from Ancient Times: Modern Reconstruction of the Greek Lyre of Hermes using $3 D$ Laser Scanning, Advanced Computer Aided Design and Audio Analysis, J. New Music Res., 44(4), 324-346.

3. Papagrigorakis, M.J., Synodinos, P.N., Antoniadis, A. Maravelakis, E., Toulas, P., Nilsson, O., Baziotopoulou-Valavani, E., (2011). Facial reconstruction of an 11-year-old female resident of 430 BC Athens, Angle Orthod., 81, 169-177.

4. Fragkos, S., Tzimtzimis, E., Tzetzis, D., Dodun, O., Kyratsis, P., (2018). 3D laser scanning and digital restoration of an archaeological find, MATEC Web of Conferences, 178, 03013.

5. Jo, Y.H., Hong, S., (2019a). Three-dimensional digital documentation of cultural heritage site based on the convergence of terrestrial laser scanning and unmanned aerial vehicle photogrammetry, ISPRS Int J Goe-Inf., 8, 53.

6. Jo, Y.H., Lee C.H., (2016). Displacement analysis of five-story stone pagoda in Geumgolsan Mountain, Jindo, using terrestrial laser scanning, Indian J Sci Techno., 9, 1-6.

7. Randhawa, J.S., Gupta, A., (2018). Virtual restoration of artefacts using 3-D scanning system, Int J Mater Mech Manuf., 6, 308-312.

8. Pavlidis, G., Koutsoudis, A., Arnaoutoglou, F., Tsioukas, V., Chamzas, C., (2007). Methods for 3D digitization of cultural heritage. J Cult Herit., 8, 9398.

9. Remondino, F., (2011). Heritage recording and $3 D$ modeling with photogrammetry and $3 D$ scanning. Remote Sens, 3, 1104-1138.

10. Remondino, F., Rizzi, A., (2010). Reality-based $3 D$ documentation of natural and cultural heritage sites-techniques, problems, and examples. Appl Geomat., 2, 85-100.

11. Bernardini, F., Rushmeier, H., (2002). The $3 D$ model acquisition pipeline, in: CGF, 21, 149-172.

12. Ikeuchi, K., Oishi, T., Takamatsu, J., Sagawa, R., Nakazawa, A., Kurazume, R., Nishino, K., Kamakura, M., Okamoto, Y., (2007). The great Buddha project: digitally archiving, restoring, and analysing cultural heritage objects, IJCV, 75(1), 189-208.
13. Levoy, M., Pulli, K., Curless, B., Rusinkiewicz, S., Koller, D., Pereira, L., Ginzton, M., Anderson, S., Davis, J., Ginsberg, J., Shade, J., Fulk, D., (2000). The digital Michelangelo project: $3 D$ scanning of large statues, SIGGRAPH, 131-144.

14. Bernardini, F., Rushmeier, H., Martin, I.M., MittleMan, J., Taubin, G., (2002). Building a digital model of Michelangelo's florentine pieta, IEEE CGA, 22(1), 59-67.

15. Miyazaki, D., Oishi, T., Nishikawa, T., Sagawa, R., Tomomatsu, T., Takase, Y., Ikeuchi, K., (2000). The great buddha project: modelling cultural heritage through observation, Proceedings of the Sixth International Conference on Virtual Systems and MultiMedia, Gifu, Japan, 138-145

16. Vrubel, A., Bellon, O.R.P., Silva, L., (2009). A $3 D$ reconstruction pipeline for digital preservation of natural and cultural assets, IEEE CVPR, 2687-2694.

Received: February 28, 2021 / Accepted: December 20, 2021 / Paper available online: December 25, 2021 (C) International Journal of Modern Manufacturing Technologies 